\title{
Data Mining for the Prediction of Heart Disease: A Literature Survey
}

\author{
P. Umasankar ${ }^{1}$ and V. Thiagarasu ${ }^{2}$ \\ ${ }^{1}$ Research Scholar, Department of Computer Science, Manonmaniam Sundaranar University, Tamil Nadu, India \\ ${ }^{2}$ Associate Professor, Department of Computer Science, Gobi Arts and Science College, Tamil Nadu, India \\ E-Mail: pus.msu2013@gmail.com
}

\begin{abstract}
The health care environment is found to be rich in information, but poor in extracting knowledge from the information. This is because of the lack of effective analysis tool to discover hidden relationships and trends in them. By applying the data mining techniques, valuable knowledge can be extracted from the health care system. Heart disease is a group of condition affecting the structure and functions of heart and has many root causes. Heart disease is the leading cause of death in the world over past ten years. Researches have been made with many hybrid techniques for diagnosing heart disease. This paper deals with an overall review of application of data mining in heart disease prediction.

Keywords: Cardio Vascular Disease, Data Mining, Feature Selection, Classification, Association Rule Mining, Clustering
\end{abstract}

\section{INTRODUCTION}

Heart is an essential organ of all living creature, which assumes a key role of propelling blood to the rest of the organs through the blood vessels of the circulatory system. Every practical issue in the heart has a straight effect on the existence of apprehensive human being; meanwhile it disturbs the further parts of the body such as lungs, brain, liver, kidney etc. Heart Diseases pronounce a range of conditions that influence the heart and remain as a major cause of death all over the globe. The clinical indications of the Heart Disease obfuscate the prediction, as it is inclined by numerous components like functional and pathologic appearance. Subsequently, this could postpone the prognosis of the illness. Hence, there is a requirement for the creation of innovative models to progress the prediction accuracy in a limited time. Disease prognosis through various elements or indications is a multi-layer problem, even that could prompt a false presumption. Like so, an endeavour is made to connect the learning and experiences of the experts and to fabricate a framework that fairly aids the diagnosing process.

Data Mining is the study of substantial datasets to extricate the hidden and formerly unidentified patterns, relationships and knowledge that are hard to investigate with conventional measurements [1]. Data mining methods are the consequence of a long method of research and product improvement. Data Mining is alienated into two assignments such as Predictive Tasks and Descriptive Tasks. Predictive Tasks forecast the estimation of an explicit attribute based on other attribute. Categorization, Regression and Deviation Deduction come under Predictive Tasks. Descriptive Tasks design that outline the connection between the data. Clustering, Association Rule Mining and
Sequential Pattern Discovery are future under Descriptive Tasks. Data Mining comprises of few steps from raw data collection to some form of new knowledge. The iterative process comprises of following stages like Data Integration, Data cleaning, Data transformation, Data Selection, Data Mining, Knowledge Representation, and Pattern Evaluation.

Medical Data Mining is a field of experiment which encompasses lot of inaccuracy and vagueness. Establishment of eminence amenities at reasonable cost is the main task confronted in the health care organization. Poor clinical decision may prompt to appalling results. Health care data is huge. Clinical decisions are frequently prepared made dependent on doctor's experience instead of on the knowledge rich data covered up in the data base. In some cases, now and again will result in errors, disproportionate medical cost which disturbs the excellence of service to the patients [2]. Medical history data involves various tests to detect the specific disease. It is conceivable to advance the benefit of Data mining in health care by retaining it as an intellectual analytical tool. The researchers in the field of medicine recognize and forecast the disease with the assistance of Data mining techniques [3].

\section{HEART DISEASE}

The initial diagnosis of a heart attack is made by a combination of clinical symptoms and characteristic electrocardiogram (ECG) changes. An ECG is a recording of the electrical activity of the heart. Confirmation of a heart attack can only be made hours later through detection of elevated creatinine phosphokinase (CPK) in the blood. CPK is a muscle protein enzyme which is released into the blood circulation by dying heart muscles when their surrounding dissolves [3]. World Health Organization in the year 2003 reported that $29.2 \%$ of total global deaths are due to Cardio Vascular Disease (CVD). By the end of this year, CVD is expected to be the leading cause for deaths in developing countries due to change in life style, work culture and food habits. Hence, more careful and efficient methods of cardiac diseases and periodic examination are of high importance [1].

\section{DATA MINING TECHNIQUES}

Data Mining is the process of extracting valid, authentic, and actionable information from large databases. Data Mining is an analytic process designed to explore data in 
search of consistent patterns and/or systematic relationships between variables, and then to validate the findings by applying the detected patterns to new subsets of data. The ultimate goal of data mining is prediction - and predictive data mining is the most common type of data mining and one that has the most direct business applications. Data mining strategies fall into two broad categories namely Supervised Learning and Unsupervised Learning. Supervised Learning methods are deployed when there exists a field or variable (target) with known values and about which predictions will be made by using the values of other fields or variables (inputs). Unsupervised Learning methods tend to be deployed on data for which there do not exist a field or variable with known values, while fields or variables do exist for other fields or variables.

\section{A. Feature Selection}

Feature selection is a process used in machine learning in which a subset of the features available from the data is selected for application of a learning algorithm. It is necessary because it is computationally not feasible to use all available features or because of problems of estimation when limited data samples are present [30]. Feature selection from the available data is vital to the effectiveness of the methods employed. Extracted features can be ranked with respect to their contribution and utilized accordingly. Existing feature selection methods for machine learning typically fall into two broad categories; those which evaluate the worth of features using the learning algorithm that is to be ultimately applied to the data, and those which evaluate the worth of features by using heuristics based on general characteristics of the data. The former is referred to as wrappers and the latter filters.

\section{B. Classification Techniques}

The classification task in machine learning is to take each instance of a dataset and assign it to a particular class [2][10]. A classification-based system attempts to classify all the patient either having heart disease or not. The challenge in this is to minimize the number of false positives and false negatives. Classification maps a data item into one of several predefined categories. These algorithms normally output "classifiers", for example, in the form of decision trees or rules. An ideal application in intrusion detection will be to gather sufficient "normal" and "abnormal" audit data for a user or a program, then apply a classification algorithm to learn a classifier that will determine (future) audit data as belonging to the normal class or the abnormal class.

\section{Clustering Techniques}

Data clustering is a common technique for statistical data analysis which is used in many fields, including machine learning, data mining, pattern recognition, image analysis and bioinformatics. It is the classification of similar objects into different groups, or more precisely, the partitioning of a data set into subsets or clusters, so that the data in each subset share some common trait which is often proximity according to some defined distance measure.

Machine learning typically regards data clustering as a form of unsupervised learning. Clustering is useful in intrusion detection as malicious activity should cluster together, separating itself from non-malicious activity [4]. Clustering is one of the most useful tasks in data mining process for discovering groups and identifying interesting distributions and patterns in the underlying data. Clustering problem is about partitioning a given data set into groups (clusters) such that the data points in a cluster are more similar to each other than points in different clusters. Clustering provides some significant advantages over the classification techniques already discussed, in that it does not require the use of a labelled data set for training.

\section{Association Rule Mining}

Association rules are if/then statements [1] that help to uncover relationships between unrelated data in a database, relational database or other information repository. Association rules are used to find the relationships between the objects which are frequently used together. Applications of association rules are basket data analysis, classification, cross-marketing, clustering, catalogue design, and lossleader analysis etc.

\section{LITERATURE REVIEW}

Nahar, Jesmin, et al., [1], examines the sick and healthy aspects which contribute to heart disease for males and females. Association rule mining, a computational knowledge method, is employed to detect these components and the UCI Cleveland dataset, an organic database, is considered alongside the three rule generation algorithms Predictive Apriori, Apriori and Tertius.

Vijiyarani, S., and S. Sudha [2] this paper investigates the classification tree methods in data mining. The objective of this paper is to explore the investigational results of the performance of various categorization techniques for a heart disease dataset. The classification tree algorithms utilized and experimented in this work are Random Forest, Decision Stump, and LMT Tree algorithm.

Gayathri, P., and N. Jaisankar [3] the review of the research associated to heart disease and furthermore the overview of numerous classifications of heart disease such as coronary artery disease, coronary heart disease, ischemic heart disease, heart failure, congenital heart disease, cardiovascular disease, hypoplastic left heart syndrome and valvular heart disease are presented in the research

Shouman, Mai, Tim Turner, and Rob Stocker [4] the analysis obtainable here is part of work to create devices to help the healthcare practitioners to detect heart disease prior in the desire for prior mediations in this preventable killer. 
The general precision of common data mining methods in heart disease findings is hard to measure from the literature. This examination explores Naïve Bayes, Decision Tree, and K-nearest Neighbour performance in the analysis of heart disease patients.

Amato, Filippo, et al., [5] this paper ANNs characterize a dominant device to aid physicians to perform analysis and different implementations. In this connection, ANNs have numerous benefits including: (i) Reduced likelihood of overlooking relevant Information (ii) The ability to process large amount of data (iii) Reduction of diagnosis time.

Persi Pamela, I., and P. Gayathri [6] A fuzzy structure is one of the delicate calculating approaches is proposed in this paper along with a data mining technique for competent analysis of coronary heart disease. However, the database has 76 attributes, only 14 attributes are initiated to be competent for CHD diagnosis as per all the distributed tests and doctors' view. Therefore, the vital attributes are taken from the heart disease database. From these attributes hard guidelines are found by retaining CART decision tree algorithm, which are then realistic to the fuzzy system. A Particle Swarm Optimization (PSO) technique is smeared for the optimization of the fuzzy membership functions where the constraints of the membership tasks are transformed to novel positions.

Chaurasia, Vikas, and Saurabh Pal [7] This research paper proposes to deliver a survey of existing methods of knowledge discovery in databases using data mining techniques which will be valuable for medical practitioners to yield successful decision. The major goal of this work is to forecast more precisely the existence of heart disease with decreased number of attributes.

Thenmozhi, K., and P. Deepika [8] the authors discovered the several decision tree algorithms like J48, ID3, C4.5 and $\mathrm{C} 5.0$ and in the classification and prediction of heart disease.

Kim, Jae-Kwon, et al., [9] This paper proposes the Fuzzy Rule-based Adaptive Coronary Heart Disease Prediction Support Model (FbACHD_PSM), which stretches comfortable reference to coronary heart disease patients. The projected model uses a mining method approved by medical experts to deliver approvals.

Seera, Manjeevan, and CheePeng Lim [10] in this paper, a hybrid intelligent system that comprises of the Fuzzy MinMax neural network, the Organization and Random Forest model, and Regression Tree is proposed, and its value as a decision support device for medical data classification is observed. The hybrid intelligent system targets to abuse the benefits of the constituent models and, at the same time, ease their impediments.

Bashir, Saba, UsmanQamar, and M. YounusJaved [11] Thegoal of the proposed research is to envisage the heart disease in a patient more precisely. The proposed structure customs common vote based novel classifier collaborated to amalgamate different data mining classifiers. UCI heart disease dataset is practiced for assessment and results.

Shabana, ASMI P., and S. Justin Samuel [12] Different data mining techniques such as Decision Tree, Naive Bayes, Association Rule and Linear Regression are practiced to envisage the heart disease. Data mining techniques in overall diagnosis realistic over all disease treatment dataset explore if hybrid data mining techniques can attain comparable (or better) results in classifying appropriate actions as that attained in the diagnosis. In this paper, the proposed work is to more precisely predict the existence of heart disease with new attributes of the disease and using association rules.

Aljaaf, A. J., et al., [13] In this study, a multi-level risk assessment of developing heart failure has been proposed, in which five levels of risks in heart failure can be predicted using C4.5 decision tree classifier. In contrast, we are enhancing the primary prediction of heart failure over concerning three core risk factors with the heart failure data set.

Bashir, Saba, UsmanQamar, and Farhan Hassan Khan [14] this research paper presents a novel classifier collaborative structure based on improved bagging approach with multiobjective weighted voting structure for analysis and prediction of heart disease. The proposed structure overwhelms the boundaries of orthodox performance by exploiting a collective of five heterogeneous classifiers: linear regression, Naive Bayes, instance-based learner, quadratic discriminant analysis and support vector machines.

Kim, Jaekwon, Jongsik Lee, and Youngho Lee [15] established model for CHD prediction must be aimed bestowing to rule-based procedures. In this study, a fuzzy logic and decision tree (classification and regression tree [CART])-driven CHD prediction model was advanced for Koreans. Datasets derived from the Nutrition Examination Survey VI (KNHANES-VI) and Korean National Health was exploited to produce the proposed method.

Joshi, Sujata, and Mydhili K. Nair [16] In this research, the classification-based data mining techniques are practiced to healthcare data. This research emphases on the forecast of heart disease using three classification techniques namely Naïve Bayes, Decision Trees, and K Nearest Neighbour.

Chadha, Ritika, et al., [17] The objective of the research is to accumulate, organize and analyse the numerous data mining techniques that have been implicit and instigated in the latest years for Heart Disease Prediction. This paper attempts to highlight blatant assessments and focus to the boons and banes of each technique. 
Choi, Edward, et al., [18] explored whether the practice of deep learning to typical temporal relations among proceedings in electronic health records (EHRs) would advance model performance in forecasting preliminary diagnosis of heart failure (HF) associated to conservative techniques that snub momentarily

Saxena, Kanak, and Richa Sharma [19] In this study, we have structured a framework that can competently discover the doctrines to forecast the risk level of patients considering the given constraint about their health. The main influence of this research is to assist a non-specialized doctor to brand accurate decision about the heart disease risk level. The guidelines engendered by the projected system are arranged as Pruned Rules, Original Rules, rules without duplicates, Sorted Rules, Classified Rules and Polish. The implementation of the structure is evaluated as far as arrangement exactness and the results reveal that the structure has astonishing prospective in expecting the coronary illness risk level all more accurately.

Goldstein, Benjamin A., Ann Marie Navar, and Rickey E. Carter [20] The purpose of this review is to demonstrate the practice of machine-learning techniques for the growth of risk prediction models. Normally offered as black box methods, most machine-learning methods are intended at unravelling specific tasks that rise in data examination that are not well addressed by distinctive regression approaches.

Miranda, Eka, et al., [21] The process of designing the method initiated by classifying the knowledge connected to the cardiovascular disease outline and the level of cardiovascular disease risk factors for adults dependent on the medical record, and scheming a mining technique model consuming a naïve Bayes classifier. Assessment of this research employed two techniques: sensitivity, accuracy, and specificity calculation as well as an assessment term with internists and cardiologists. The features of cardiovascular disease are recognized by its principal risk factors. Those aspects are the level of lipids in the blood, diabetes mellitus, kidney function, and coronary artery function. Class markers were dispensed in relation to the standards of these factors: risk level 1, risk level 2 and risk level 3.

Chadha, Ritika, and Shubhankar Mayank [22] This paper endeavours to elicit the organization and execution of these techniques Decision Tree, Artificial Neural Networks, and Naive Bayes and strain upon the effects and inference persuaded on the base of exactness and time intricacy. By far, the opinions expose that Artificial Neural Networks outstripped Decision Tree and Naive Bayes.

Singh, Garima, et al., [23] The central objective of this investigation is to cultivate a archetype Health Care Prediction System using, Naive Bayes. The System will determine and abstract hidden data associated to diseases (heart attack, cancer and diabetes) from a chronological heart disease database. It will response for the problematical queries for analysing sickness and consequently assist healthcare practitioners to practice intellectual clinical selections which prehistoric call support systems cannot do with.

Pouriyeh, Seyedamin, et al., [24] This paper aims to probe and link the exactness of dissimilar data mining classification structures and their amalgamations over Ensemble Machine Learning Techniques for predicting heart disease.

Samuel, Oluwarotimi Williams, et al., [25] In this study, the frequently used $13 \mathrm{HF}$ attributes were measured and their contributions were firm by an knowledgeable cardiac clinician. And Fuzzy analytic hierarchy process (Fuzzy_AHP) technique was cast-off to calculate the universal weights for the attributes constructed on their distinct contribution. At that time, the global weights that epitomize the contributions of the attributes were instigated to train an ANN classifier for the prediction of HF risks in patients.

Tripoliti, Evanthia E., et al., [26] The goal of this paper is to show the state-of-the-art of the machine learning methodologies practiced for the evaluation of heart failure. More precisely, models forecasting the existence, approximating the subtype, evaluating the severity of heart failure and forecasting the existence of confrontational actions, such as re-hospitalizations, destabilizations, and mortality are presented.

Acharya, U. Rajendra, et al., [27] Electrocardiogram is broadly employed to diagnose the congestive heart failure $(\mathrm{CHF})$. It is the principal non-invasive diagnostic device that can escort in the administration and follow-up of patients with CHF. Heart rate variability (HRV) signals which are nonlinear in nature hold the hidden signatures of numerous cardiac diseases. As a result, this paper recommends a nonlinear method, empirical mode decomposition (EMD), for a mechanized identification and classification of standard and CHF using HRV signals. In this research, HRV signals are endangered to EMD to acquire intrinsic mode functions (IMFs).

Seah, Jarrel CY, et al., [28] To examine Generative Visual Rationales (GVRs) as a device for envisaging neural system knowledge of chest radiograph features in congestive heart failure (CHF).

Kumar, Priyan Malarvizhi, and Usha Devi Gandhi [29] The data generation speed of IoT sensor devices is very extraordinary. As a result, the size of data produced from the IoT-based health monitoring system is also very high. So as to overwhelm this problem, this paper suggests mountable three-tier architecture to store and method such massive volume of wearable sensor data. Tier-1 emphases on collection of information from IoT wearable sensor devices. Tier-2 customs Apache HBase for loading the large size of wearable IoT sensor data in cloud calculating. 
Jain, Divya, and Vijendra Singh [30] This paper grants a survey on the exploitation of feature selection and classification techniques for the analysis and forecast of chronic diseases. Ample selection of features shows a substantial role for augmenting accuracy of classification systems. Dimensionality reduction supports in enlightening global performance of machine learning algorithm.

Paul, Animesh Kumar, et al., [31] In this paper, we have industrialised a computed fuzzy diagnostic system based on genetic algorithm (GA) and a modified dynamic multiswarm particle swarm optimization (MDMS-PSO) for predicting the risk level of heart disease. Our planned fuzzy diagnostic system (FS) works as follows: i) Effective attributes are selected through statistical methods such as Correlation coefficient, R-Squared and Weighted Least Squared (WLS) method ii)Pre-process the data sets iii) Weighted fuzzy rules are formed other basis of selected attributes using GA, iv) MDMS-PSO is employed for the optimization of membership functions (MFs) of FS, v) Build the ensemble FS from the generated fuzzy knowledge base by fusing the different local FSs.

Manogaran, Gunasekaran, R. Varatharajan, and M. K. Priyan [32] Multiple Kernel Learning with Adaptive NeuroFuzzy Inference System (MKL with ANFIS) based deep learning method is proposed in this research for heart disease diagnosis. The anticipated MKL with ANFIS based deep learning method tails two-fold approach. MKL technique is cast-off to distribute constraints between normal individuals and heart disease patients.

\section{CONCLUSION}

The most important and difficult task in medicine is Medical diagnosis. The problem here is detecting a disease from several factors or symptoms, since it may lead to false assumptions with unpredictable results. Heart disease prediction is a major challenge in the healthcare industry. Instead of going for a number of tests, predicting heart disease with a smaller number of attributes is challenging task in Data Mining. Existing literature shows that Classification task in Data Mining plays a vital role in heart disease prediction when compared with Clustering, Association Rule and Regression.

\section{REFERENCES}

[1] Nahar, Jesmin, et al., "Association rule mining to detect factors which contribute to heart disease in males and females", Expert Systems with Applications, Vol. 40, No. 4, pp. 1086-1093, 2013.

[2] Vijiyarani, S., and S. Sudha, "An efficient classification tree technique for heart disease prediction", International Conference on Research Trends in Computer Technologies (ICRTCT-2013) Proceedings published in International Journal of Computer Applications (IJCA)(0975-8887). Vol. 201, 2013.

[3] Gayathri, P., and N. Jaisankar, "Comprehensive study of heart disease diagnosis using data mining and soft computing techniques", 2013.

[4] Shouman, Mai, Tim Turner, and Rob Stocker, "Integrating clustering with different data mining techniques in the diagnosis of heart disease", J. Comput. Sci. Eng, Vol. 20, No. 1, 2013.
[5] Amato, Filippo, et al., "Artificial neural networks in medical diagnosis", pp. 47-58, 2013.

[6] Persi Pamela, I., and P. Gayathri, "A fuzzy optimization technique for the prediction of coronary heart disease using decision tree", 2013.

[7] Chaurasia, Vikas, and Saurabh Pal, "Data mining approach to detect heart diseases", 2014.

[8] K. Thenmozhi, and P. Deepika, "Heart disease prediction using classification with different decision tree techniques", International Journal of Engineering Research and General Science, Vol. 2, No. 6, pp. 6-11, 2014.

[9] Kim, Jae-Kwon, et al., "Adaptive mining prediction model for content recommendation to coronary heart disease patients", Cluster computing, Vol. 17, No. 3, pp. 881-891, 2014.

[10] Seera, Manjeevan, and CheePeng Lim, "A hybrid intelligent system for medical data classification", Expert Systems with Applications, Vol. 41, No. 5, pp. 2239-2249, 2014.

[11] Bashir, Saba, UsmanQamar, and M. YounusJaved, "An ensemblebased decision support framework for intelligent heart disease diagnosis", Information Society (i-Society), 2014 International Conference on. IEEE, 2014.

[12] Shabana, ASMI P., and S. Justin Samuel, "An analysis and accuracy prediction of heart disease with association rule and other data mining techniques", Journal of Theoretical and Applied Information Technology, Vol. 79, No. 2, pp. 254-60, 2015.

[13] A. J. Aljaaf, et al., "Predicting the likelihood of heart failure with a multi-level risk assessment using decision tree", Technological Advances in Electrical, Electronics and Computer Engineering (TAEECE), 2015 Third International Conference on IEEE, 2015.

[14] Bashir, Saba, UsmanQamar, and Farhan Hassan Khan, "BagMOOV: A novel ensemble for heart disease prediction bootstrap aggregation with multi-objective optimized voting", Australasian physical \& engineering sciences in medicine, Vol. 38, No. 2, pp. 305-323, 2015.

[15] Kim, Jaekwon, Jongsik Lee, and Youngho Lee, "Data-mining-based coronary heart disease risk prediction model using fuzzy logic and decision tree", Healthcare informatics research, Vol. 21, No. 3, pp. 167-174, 2015.

[16] Joshi, Sujata, and Mydhili K. Nair, "Prediction of heart disease using classification-based data mining techniques", Computational Intelligence in Data Mining-. Springer, New Delhi, Vol. 2, pp.503511, 2015.

[17] Chadha, Ritika, et al., "Application of data mining techniques on heart disease prediction: a survey", Emerging Research in Computing, Information, Communication and Applications. Springer, New Delhi, pp. 413-426, 2016.

[18] Choi, Edward, et al.,, "Using recurrent neural network models for early detection of heart failure onset", Journal of the American Medical Informatics Association, Vol. 24, No. 2, pp. 361-370, 2016.

[19] Saxena, Kanak, and Richa Sharma, "Efficient Heart Disease Prediction System",Procedia Computer Science, Vol. 85, pp. 962969, 2016.

[20] Goldstein, A. Benjamin Ann Marie Navar, and Rickey E. Carter, "Moving beyond regression techniques in cardiovascular risk prediction: applying machine learning to address analytic challenges", European heart journal, Vol. 38, No. 23, pp. 1805-1814, 2016.

[21] Miranda, Eka, et al., "Detection of cardiovascular disease risk's level for adults using naive Bayes classifier", Healthcare informatics research, Vol. 22, No. 3, pp. 196-205, 2016.

[22] Chadha, Ritika, and ShubhankarMayank, "Prediction of heart disease using data mining techniques", CSI transactions on ICT, Vol. 4.2-4, pp. 193-198, 2016.

[23] Singh, Garima, et al., "Heart disease prediction using Naïve Bayes", International Research Journal of Engineering and Technology (IRJET) e-ISSN: 2395-0056, 2017.

[24] Pouriyeh, Seyedamin, et al., "A comprehensive investigation and comparison of Machine Learning Techniques in the domain of heart disease", Computers and Communications (ISCC), IEEE Symposium on IEEE, 2017.

[25] Samuel, Oluwarotimi Williams, et al., "An integrated decision support system based on ANN and Fuzzy_AHP for heart failure risk prediction”, Expert Systems with Applications, Vol. 68, pp. 163-172, 2017.

[26] Tripoliti, E. Evanthia et al., "Heart failure: diagnosis, severity estimation and prediction of adverse events through machine learning 
techniques", Computational and structural biotechnology journal, Vol. 15, pp. 26-47, 2017.

[27] Acharya, U. Rajendra, et al., "Application of empirical mode decomposition (EMD) for automated identification of congestive heart failure using heart rate signals", Neural Computing and Applications, Vol. 28, No. 10, pp. 3073-3094, 2017.

[28] Seah, C. Y. Jarrel, et al., "Chest radiographs in congestive heart failure: visualizing neural network learning",Radiology (2018): 180887, 2018.

[29] Kumar, PriyanMalarvizhi, and Usha Devi Gandhi, "A novel three-tier Internet of Things architecture with machine learning algorithm for early detection of heart diseases",Computers \& Electrical Engineering , Vol. 65, pp. 222-235, 2018.
[30] Jain, Divya, and Vijendra Singh, "Feature selection and classification systems for chronic disease prediction: A review",Egyptian Informatics Journal, 2018.

[31] Paul, Animesh Kumar, et al., "Adaptive weighted fuzzy rule-based system for the risk level assessment of heart disease", Applied Intelligence, Vol. 48, No. 7, pp. 1739-1756, 2018.

[32] Manogaran, Gunasekaran, R. Varatharajan, and M. K. Priyan, "Hybrid recommendation system for heart disease diagnosis based on multiple kernel learning with adaptive neuro-fuzzy inference system", Multimedia tools and applications, Vol. 77, No. 4, pp. 4379-4399, 2018. 